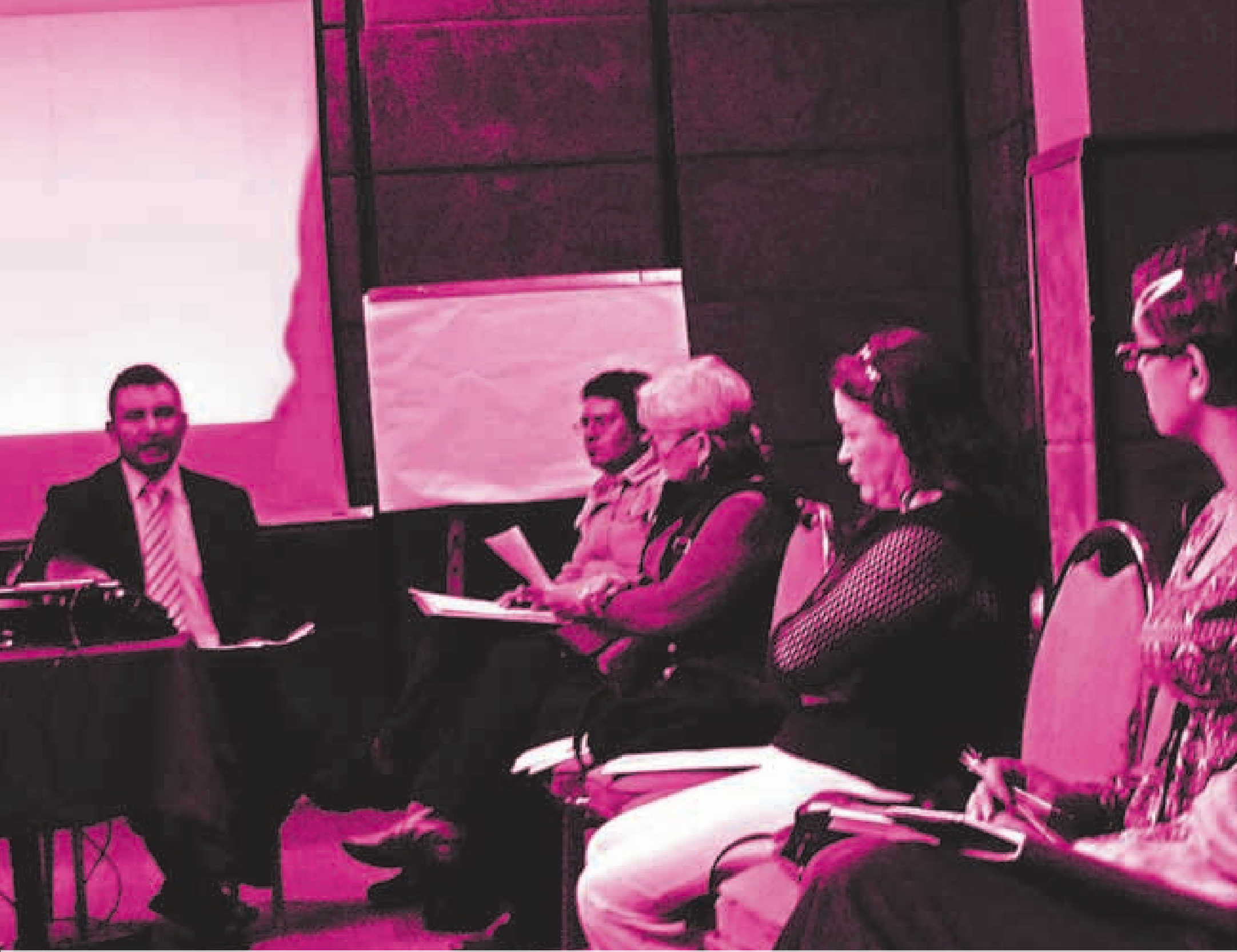

\title{
Formar docentes en tiempos de equivalencias generalizadas
}

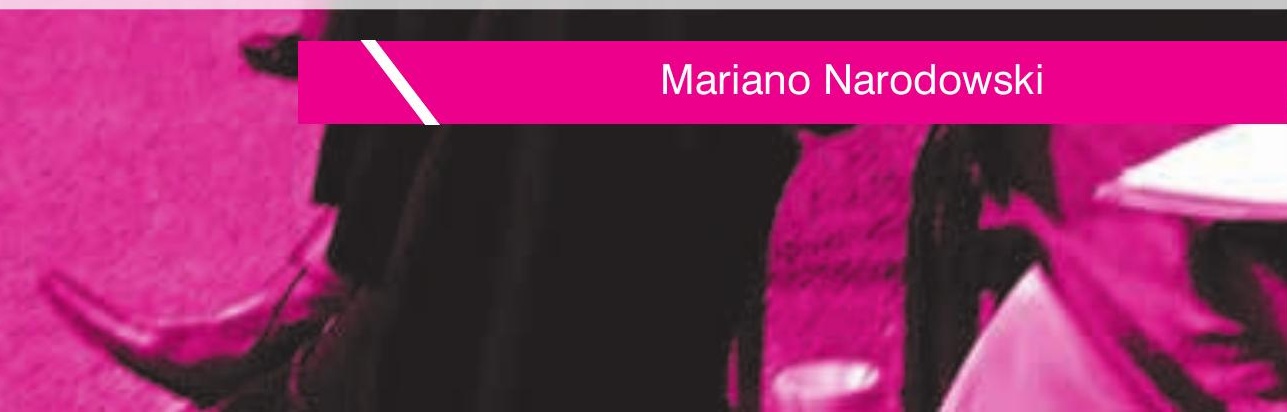




\section{Resumen}

Este artículo analiza los cambios culturales ocurridos en la situación de la infancia y la adultez en los últimos cincuenta años y sus consecuencias en el campo de la educación escolar y, especialmente, de la formación docente.

En la primera parte, y en base a los clásicos trabajos antropológicos de Margaret Mead, el artículo explica la naturaleza histórica de la infancia y de la adultez y las condiciones culturales que daban sustento a los vínculos asimétricos que entre ellos se establecían, advirtiendo que las instituciones escolares de la modernidad funcionaban a partir de estas características y estas condiciones.

En la segunda parte de este trabajo, se desarrollan las transformaciones operadas en el seno de la condición posmoderna de la cultura para tratar de comprender cómo las asimetrías van perdiendo su legitimidad y con ellas el posicionamiento socialmente naturalizado de la función adulta. Se explica que relaciones desjerarquizadas ocupan el lugar de las asimetrías tradicionales y que, por ende, se materializan en una generalización de los vínculos basado en posiciones crecientemente equivalentes.

Finalmente, el artículo advierte respecto de las efectos de esta situación en la formación docente. Se postula la necesidad de enfoques basados en escenarios de crisis recurrentes y en la necesidad de perfiles de educadores flexibles, atentos a las oportunidades que se presentan para la construcción de asimetrías contingentes y se sugieren caminos estratégicos para ese logro.

\section{Palabras clave}

Formación docente, infancia, fin de la infancia, posmodernidad, culturas posfigurativas.

\section{Abstract}

This article analyses the cultural changes that affected childhood and adulthood in the last fifty years and their consequences in both the fields of education and teacher education in particular.

In the first part, and based on the anthropological works of Margaret Mead, the article explains the historic nature of childhood and adulthood and the cultural conditions that supported the asymmetrical relationships established between them. It also points out that modern school could actually work thanks to these characteristics and conditions.

In the second part of the work, the transformations that took place in the core of the culture's postmodern condition are developed to attempt at understanding how the asymmetries gradually lose their legitimacy. As a consequence, the socially naturalized position of the adults is eventually lost as well. Non-hierarchical relationships take the place of the traditional asymmetries and, as a result, the relationships become generalized based on increasingly equivalent positions.

Finally, the article warns about the effects of the situation described on teacher education. There is a need for approaches based on recurrent crises scenarios and flexible educators. These teachers should be aware of the opportunities that may arise for the construction of contingent asymmetries. Strategic paths to this achievement are suggested as well.

Key words

Teacher education, childhood, end of childhood, postmodernity, postfigurative cultures.

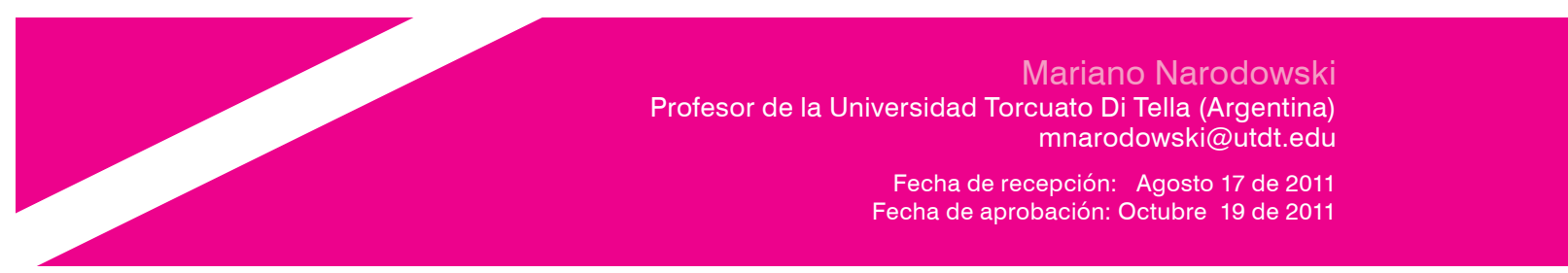




\section{Las asimetrías fundantes}

Hacia 1970, en plena ebullición de los movimientos sociales juveniles en diferentes países del mundo occidental, proceso que tuviera en el famoso mayo del 68 francés su máximo emergente político y simbólico-, se publica el libro Cultura y Compromiso de la eminente antropóloga Margaret Mead (Mead, 2006). La alusión a la época es importante ya que el momento en el que el libro se edita no era irrelevante: esta obra de Mead se dedicó a indagar el problema de las rupturas generacionales (que era un tema central del debate de los sesenta y de los sesenta) y las condiciones sociales y culturales que deben acaecer para que estas rupturas pudieren desarrollarse.

El enorme valor de este libro se basa no solamente en la profundidad de su análisis sino, especialmente, en lo estimulante de sus categorías, ya que, más allá de las cuatro décadas pasadas desde su publicación, de la desaparición del entorno social y cultural que lo viera nacer y de la probable decepción que trajo aparejada el inicial entusiasmo sesentaiochista, el trabajo de Mead resulta una cantera inagotable de pensamiento sobre infancias y juventudes, que trasciende enormemente la época en la cual y -posiblemente- para la cual fuera escrito.

En el entramado de generaciones tras generaciones, existen diferencias respecto de cómo grandes y chicos articulan entre sí sus propias existencias. El caso de la cultura en la que existe una infancia subordinada a la guía y al cuidado adulto es la que Margaret Mead denomina "cultura posfigurativa" (1986: 29 y ss).

En estas sociedades, los chicos reciben desde el inicio de sus vidas el conocimiento y la protección de sus mayores. $Y$ ese saber hacer para la vida que reciben ya desde el inicio, les sirve básicamente para su edad adulta, toda vez que los cambios que habrán reproducirse en la sociedad en cuestión serán lentos, poco significativos y casi imperceptibles: lo que los adultos trasmiten desde la más tierna infancia humana es lo que, con certeza, tendrá vigencia para toda la vida.

Esta evidente legitimidad de la acción adulta propia de la cultura posfigurativa se resume en una práctica educacio- nal nuclear: "seguir el ejemplo". Los más chicos siguen el ejemplo de los mayores y así garantizarán el éxito de sus acciones ulteriores ya que los siguiendo la guía, todo problema que se presente habrá de resolverse satisfactoriamente ya que en estas culturas no hay (o hay muy pocos) situaciones radicalmente nuevas. La educación, por tanto, no es otra cosa que la consecuencia de la acumulación lineal y sin rupturas de experiencias y conocimientos por parte de las personas a lo largo de sus vidas: en las culturas posfigurativas, el monto de la experiencia social acumulada y atesorada a lo largo del tiempo permite tener una cosmovisión lúcida de la realidad lo que brinda una base razonable para tomar decisiones adecuadas relativas a un futuro que no habrá de ser demasiado diferente que el pasado.

Así. En estas culturas de carácter posfigurativo, la experiencia acumulada por los mayores es la fuente incuestionable de la legitimidad adulta para comprender y gobernar la vida, como así también el instrumento social utilizado para reproducirla y reforzarla. Es el paso del tiempo el que, genera las condiciones para e su acumulación por lo que una mayor edad de una persona la torna más experiente y por ende con una, mayor legitimidad para decidir por sí y por los otros aún incapaces de decidir por sí mismos: los niños.

Esto no significa que las culturas posfigurativas sean absolutamente armónicas o exentas de conflictos. Para Margaret Mead, en estas culturas suele ocurrir que en determinado momento del crecimiento de los más jóvenes, estos se sienten con derecho a cuestionar la autoridad de sus mayores y suelen hacerlo con dureza y ambición. Sin embargo esta etapa se supera puesto que, para la investigadora, la propia identidad cultural se refuerza con amenazas y castigos a los desafiantes que serán finalmente refrendados por la persistencia de la cultura desafiada. (Mead, 2006: 141 y ss).

En las culturas posfigurativas la subordinación de las jóvenes generaciones a las generaciones mayores termina por ser socialmente naturalizada: los más viejos saben

1. Como toda gran obra, Cultura y Compromiso de Margaret Mead es hija legítima de su época, lo que explica el entusiasmo que la autora deja traslucir por los movimientos juveniles. De esta manera se explica su llamado final, cuando profetiza con entusiasmo revolucionario, juvenil y -por qué no- un poco hippie: "El futuro es ahora". 
más y pueden más, por el solo hecho de ser más viejos. Los más viejos vivieron más y en ese sentido, toda nueva experiencia por la que atraviesan las nuevas generaciones será considerada como una experiencia necesariamente ya vivida por un mayor: no hay nada nuevo bajo el sol y la imitación de los modelos adultos es la fuente de maduración para las jóvenes generaciones. (Narodowski, 1999).

Como puede fácilmente inferirse la noción de tiempo en estas culturas posfigurativas habrá de tener dos elementos centrales: la linealidad la capacidad de ser acumulado: la antigüedad garantiza el saber y cuanto más viejo se es, más se sabe.

Para Margaret Mead, el elemento distintivo de la cultura postfigurativa es la existencia de al menos tres generaciones que, al mismo tiempo, dan a la cultura existente por supuesta y para así conseguir que los niños -las nuevas generaciones- incorporen todo lo que se les enseña a lo largo de su formación. La impugnación a este canon tendrá posibilidades de éxito sólo si la sociedad necesita desplazarse o si es influida -"contaminada"- por otra cultura que asume un carácter invasor. Mientras esto no acontezca, se trata de sociedades basadas en el respeto y en la devoción a sus viejos; sociedades que habrán de venerar a sus mayores y a sus atributos. Sociedades que formarán, cuidarán y protegerán a los niños de acuerdo a los preceptos establecidos por los más viejos.
Es evidente que en nuestra cultura posfiguratuiva occidental, la escuela ha sido el ámbito esencial que remitía a formación y protección de la infancia. En una cultura en las que se confunde el saber con la traición, las escuela juega el rol naturalizado de su articulación. Desde los albores de la modernidad, la escuela ha sido institucionalizada como el espacio por antonomasia para fijar a los niños para que las viejas generaciones puedan educarlos adecuadamente (Narodowski, 1994).

Bajo el continente conceptual y emocional que remite al cuidado o a la protección de quienes denominamos menores de edad la escuela protege a niños y adolescentes de otros que pudieren aprovecharse de su heteronomía (es decir, de la incapacidad que los adultos le adjudican para construir una ley propia) pero sobre todo los cuidamos de si mismos, de su incapacidad para tomar decisiones que fortalezcan un destino posterior e, incluso menos ambiciosamente, que directamente pongan en juego su propia sobrevivencia física. A la vez, la escuela permite a los menores un acercamiento gradual y secuenciado a la cultura adulta y favorece aprendizajes que permitirán convertirse en un adulto con capacidad de autogobierno de acuerdo a las reglas de esta cultura.

La característica saliente que nuestras sociedades occidentales les habían atribuido a los menores de edad a raíz de sus limitaciones y de su desconocimiento, era la inocencia. ${ }^{2} \mathrm{Al}$ contrario de la vieja tradición que veía en

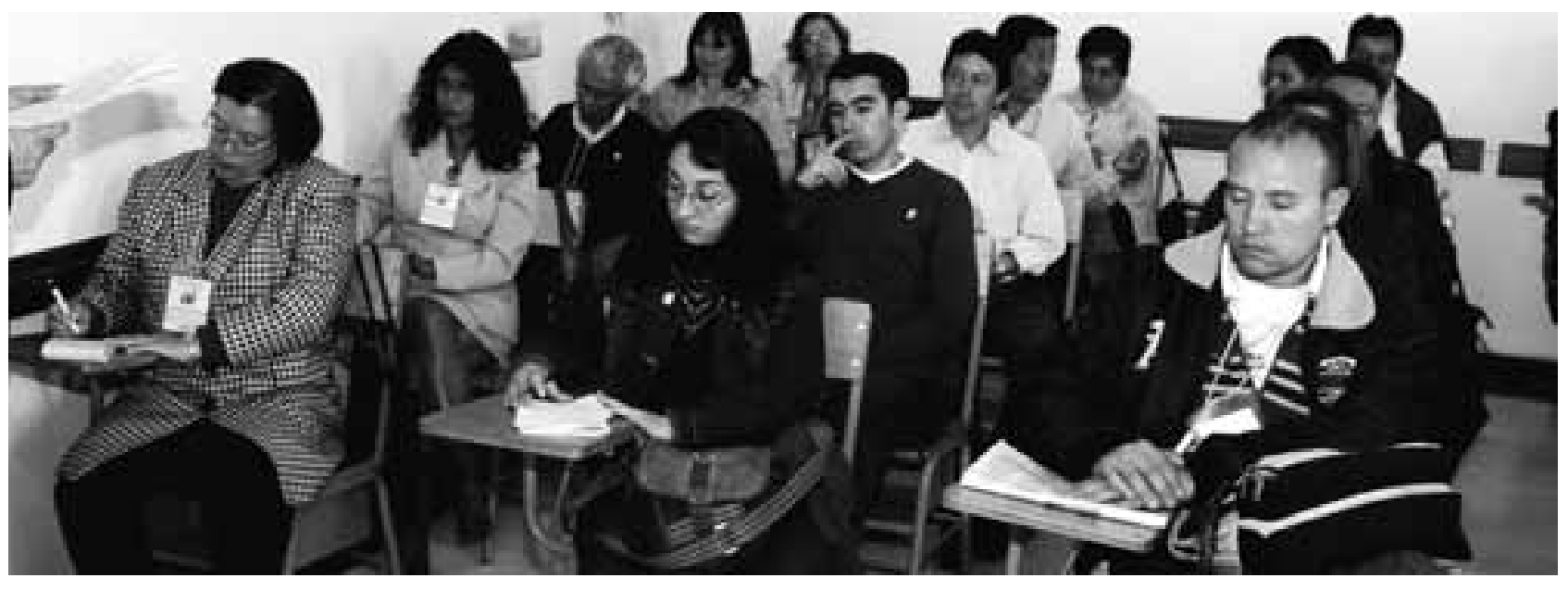

2. Para un análisis reciente del concepto kantiano de "minoría de edad" ys sus consecuencias en la conformación del moderno proceso de infantilización véase Narodowski (2011a) 
un niño no más que un adulto falto de sus habilidades, la modernidad promueve una visión radicalmente diferente: los niños están limpios, su obrar no obedece a la maldad puesto que su carácter heterónomo no provee el don de reconocer valores determinados. Si la infancia era, para nuestras sociedades, la edad de la inocencia, la adultez estaba ahí para cuidar, proteger y guiar.

La lógica vincular, en una cultura posfigurativa, entre el niño y adulto es siempre y necesariamente de carácter asimétrico ya que las responsabilidades de cada parte lejos de ser equivalentes son opuestas: el adulto es responsable por el niño porque en este se haya la incapacidad moral de llevar adelante su vida por sus propios medios, sin ponerla en peligro y sin hacer peligrar a otros. Como es sabido, la escuela de la modernidad reproducía íntegramente esta ecuación: el docente ocupaba el lugar del adulto que sabe y el alumno es definido en forma infantilizada: obediente, dependiente, heterónomo y ocupante del lugar de no-saber.

Por asimetría, se entiende aquí una relación aritmética en la cual al aplicar una regla o una operación, el resultado obtenido difiere en cada uno de los elementos de la relación. En el caso de la relación adulto-niño/adolescente, ese resultado muestra propiedades significativamente diferenciadas entre unos y otros. Entre los términos de la relación asimétrica existe desigualdad aunque no necesariamente desequilibrio: la vinculación de carácter asimétrico permite vínculos menos homogéneos que la simetría porque refieren al orden de la alteridad pero dinámicamente posibles de acuerdo a la modalidad de relación asimétrica que se erija

La asimetría adulto-niño implica que el vínculo entre uno y otro no es entre iguales, sino todo lo contrario. Por ser "el ejemplo", el adulto mantiene responsabilidades que el niño no posee, aunque este posea las suyas, siempre diferenciadas de las del adulto. En las relaciones sociales de la educación escolar esto puede vislumbrarse sin demasiadas limitaciones ya que es del maestro la responsabilidad final del proceso educacional y a él le corresponden las decisiones acerca de los dispositivos de funcionamiento escolar.

Formarse para llegar a ser un maestro, implicaba asumir como propias diferentes saberes, métodos y técnicas en un escenario dado por la asimetría, lo que construía una autoridad docente "natural", legitimada por su mismo origen docente. Este escenario asimétrico preveía que, en todo caso, los problemas venían de la mano del uso abusivo de la posición de autoridad y llevase la asimetría a una lógica de dominio, castigos corporales, etc.

\section{Las equivalencias generalizadas}

Como pudo colegirse en párrafos los precedentes, el uso de tiempos verbales pretéritos para aludir a la cultura posfigurativa, a la modernidad escolar y a la propia autoridad adulta del docente, dejan entrever la idea de que buena parte de las prácticas culturales que daban sustento al ejerció docente de la modernidad han sido sustituidas por otras prácticas y otros parámetros culturales.

Si bien algunos de estos postulados aún son materia de controversia (Buckingham, 2010) es posible al menos conjeturar que nuestro tiempo presente ya no parece estar signado por el imperio de la experiencia adulta en una cultura epocalmente estable o con cambios mínimos, tal como ocurría en la cultura posfigurativa. Por el contrario, y siempre siguiendo a Margaret Mead (2006), en la actual cultura dominante la vejez e incluso la adultez parecen haber pasado a un poco decoroso segundo plano y la exaltación de lo nuevo, de lo juvenil, e incluso de lo infantil está al orden del día.

Al contrario de las culturas posfigurativas, los cambios vertiginosos en un determinado escenario social son los que establecen una forma diferente en el intercambio intergeneracional: serán ahora los niños y los jóvenes los portadores de bienes culturales valiosos ya que su dominio de acciones y discursos fue configurado en la nueva situación.

Nuestra cultura de cambios constantes y violentos, expresa la necesidad de nuevas experiencias y reivindica infancias y adolescencias antes desechadas por su falta de un juicio atinado sobre la realidad: solamente aquello que se formaron en la vorágine de los cambios son los que pueden interactuar en ellos. A los otros, a los que pertenecen a generaciones anteriores, solo les queda intentar ajustarse a lo nuevo siguiendo la huella dejada por los más jóvenes: la ecuación parece haberse invertido y con ella la lógica asimétrica que la sustentaba. 
Esta es la característica de los tiempos actuales como lo han señalado diferentes autores adscriptos a diferentes posturas teóricas ${ }^{3}$. Nosotros mismos, hace ya muchos años, hemos advertido que en campo de la pedagogía y

sus disciplinas conexas es necesario revisar el significado moderno de "infancia" (Narodowski y Baquero, 1994), ya que su desarrollo parece más bien propio de culturas posfigurativas, lo que hace necesaria su reconceptualización en el contexto de culturas prefigurativas.

En otras palabras, estos cambios hacen que las infancias/adolescencias ya no sean las mismas ${ }^{4}$. La vieja visión de un niño dependiente, obediente y heterónomo elaborada meticulosamente en los últimos quinientos años es cuestionada por la sobrevalorización de la infancia, de lo joven; por la exaltación -incluso- de la inexperiencia de las generaciones más jóvenes. Ser joven, -ser niño o adolescente- ya no supone una carencia que va a ser superada por la correcta acción educativa de los adultos que se va a brindar a través del paso del tiempo y especialmente de la escuela. Infancia, adolescencia, juventud, constituyen seductores caracteres no solamente en ellos sino, y esto es lo más importante, en los adultos que ahora intentan lograr una fisonomía exterior, un lenguaje, unos gustos estéticos asimilables a los de los más jóvenes. Esa es su forma de seguir vigentes.

La cultura prefigurativa que habitamos edifica un progresivo desdén hacia la adultez. Como ya analizáramos en otro lado (Narodowski, 2011b), esta situación fue magistralmente sintetizada por la producción hollywoodense "Big" de 1988, conocida en el mundo castellano parlante como "Quisiera ser grande" (Marshall, 1988). En esta comedia romántica, el niño Josh Baskin, cansado de los infortunios infantiles y de los términos que se le imponen a su corta edad, desea "ser grande" y el Genio de un viejo juego de un parque de diversiones le otorga su deseo. Josh adulto, interpretado por un muy joven Tom Hanks, mantiene en su cuerpo de adulto su misma cognición infantil y sólo con ella logra triunfar en los negocios y seducir a una bella -y no poco competitiva- compañera de trabajo: su desfachatez, su candor, su simplicidad y especialmente su inexperiencia son muy valoradas en el nuevo mundo adulto, en contraposición a las posturas tradicionalistas y rígidas de los "viejos" adultos a los que se enfrenta en su nuevo trabajo. En la película está clara la lógica de los prefigurativo: pensar y actuar como un niño es cool.

Los inconvenientes para Josh inician cuando se toma demasiado en serio su nuevo rol de grande. Por un lado, sus acciones y sus ideas comienzan a perder preeminencia porque ante tanto contacto con adultos reales empieza a comportarse como uno más entre ellos y a opinar como un adulto. Sus comentarios, por lo tanto, ya no son frescos e innovadores... empiezan a oler a viejo. Por otro lado, Josh se da cuenta que el mundo adulto no es realmente tan encantador como él había imaginado cuando niño; que mucho más interesante lo es su infancia perdida y, por eso, decide retrotraer el hechizo y volver a ser chico.

Está claro que nuestra cultura va perdiendo sostenidamente las viejas diferencias entre grandes y chicos y ser adulto ya no parece un valor que concite legitimidad y respeto. La tendencia de nuestras sociedades es a la creciente desjerarquización, una horizontalización que implica el opuesto de las jerarquías asimétricas de antaño (Sennett, 2007). En este panorama, los agentes sociales son reconocidos como portadores de saberes legítimos posibles de ser intercambiados por medio de un aplanamiento desjerarquizado de las relaciones sociales, especialmente de las relaciones sociales de la educación. Donde antes había provisión asimétrica de conocimientos ahora puede hallarse el imperio de la pura intercambiabilidad. Donde antes las jerarquías de edad y de conocimientos emplazaban al otro al lugar de la dependencia y la heteronomía (a la infantilización) hoy reina la regulación de una suerte de simetría que, aunque sin saberlo, proclama una revalorización de relaciones prácticamente sindiásmicas.

En ese sentido, más que jerarquías son las equivalencias las que se han universalizado y la autoridad ya no lo es por la marca de su origen sino, en el mejor de los casos, habrá de legitimarse por la significatividad que encierra su ejercicio: una legitimidad siempre contingente, situacional y evidentemente pasajera.

3. En los extremos del debate, podemos citar a Daniel Bell (1996) y a Jürgen Habermas (1980).

4. Una visión más extensa de esta cuestión ya la hemos planteado en Mariano Narodowski (1999 y 2005). 


\section{La reconfiguración de la docencia}

Este nuevo escenario tiene enormes consecuencias en el campo de la educación escolar. La esfera del saber se ha divorciado de la esfera de la tradición por lo que el que enseña no es embajador de ninguna tradición preexistente que tenga valor en sí misma. Sólo perviven los ritos escolares institucionalizados desde los inicios (la centralidad del docente en la clase, su rol en la enseñanza, su capacidad de evaluar y calificar, su obligación de mantener el orden, etc) pero con esas rutinas, y sólo con ellas, no alcanza para que la acción docente cobre significatividad cultural.

Sí es posible que docentes y alumnos mantengan relaciones asimétricamente coyunturales, pero las mismas ya no se dan por defecto, por la sola presencia adulta, sino que solo pueden ser establecidas por algún maestro experto con capacidad para articularlas. Por cierto, articulación igualmente porosa y situacional, que difícilmente se haga crónica en una institución escolar (Wheller et. al., 2008).

Si el maestro de la modernidad posfigurativa era una suerte de embajador de la tradición y a la vez un espejo en el cual los niños debían mirarse para llegar a ser adultos de bien (de bien para esa sociedad moderna y posfigurativa) el maestro de la posmodernidad prefigurativa ya no tiene tradiciones a las que representar y, por el contrario, su propia entidad adulta no tiene -en sí misma- ninguna relevancia representativa desde el punto de vista de su legitimidad social. Poco vale por ser adulta (y en muchos contextos sociales está, incluso, desvalorizada por serlo).

¿Cuáles son, entonces, los desafíos que presenta la formación de docentes en el contexto de estas transformaciones radicalizadas que modificaron un viejo escenario escolar amparado en una cultura con centralidad adulta y lo convirtieron en uno en el que dicha centralidad sigue siendo ritual escolar cotidiano aunque ya sin el cobijo de la cultura posfigurativa y, por el contrario, víctima de un fuerte cuestionamiento a la autoridad adulta?

La respuesta no solamente que no es sencilla sino que requiere de no pocos reparos a fin de que los cambios futuros -fácilmente predecibles en su ocurrencia mas no es su contenido-, puedan ser resignificados en una matriz explicativa con capacidad de proyectar y de brindar a los futuros docentes, y especialmente a quienes se ocupan de su formación, un conjunto instrumental y conceptual que los ayude a reconceptualizar el nuevo escenario y sus constantes mutaciones.

En esta línea, se habrán de postular dos ejes conceptuales que, retomando evidencias de investigaciones recientes, permiten avanzar en la comprensión del perfil del futuro docente y las diferentes alternativas de su formación.

En primer término, es menester aclarar que los futuros docentes son ya portadores de valores culturales prefigurativos y su experiencia de vida está ya bien arraigada en los cambios que este escenario posmoderno hubo de instalar. El estupor inicial, el primigenio desconcierto o la perplejidad frente a la nueva situación era potestad absoluta de quienes habían procesado su propia formación como docentes durante la vieja escena. Por el contrario, los docentes hoy en formación son ellos mismos protagonistas de los cambios que a las generaciones anteriores generaban perplejidad. Estos nuevos docentes no habrán de asombrarse por el imperio del canon cultural prefigurativo y las situaciones que este genera forman o parte de su sentido común de época.

En segundo lugar, la constatación evidente de que la institución escolar mantiene sus ritos posfigurativos no solamente presupone un escenario de crisis constantes sino que anticipa el hecho de la lógica asimétrica, a pesar de todo, es la única que puede funcionar en semejante contexto. Este diagnóstico se deriva dos consecuencias que merecen la pena al menos ser enunciadas.

La primera consecuencia, implica un cambio de enfoque respecto de la imagen del educador en el contexto escolar. Como se ha advertido en otros trabajos (Narodowski, 1994), la tradición pedagógica moderna aspiraba a montar un escenario ordenado y armónico por medio de la acción pedagógica de un docente que ejerce un método didáctico racional. Hoy, esa pretensión resulta cuanto menos ambiciosa y omnipotente a la luz de sus pobres resultados, y su persistencia entre educadores y pedagogos no puede ser explicado de otra forma que por medio de una remisión neurótica (y neuróticamente repetitiva) como respuesta ineficaz frente a las constantes frustraciones que la realidad de las aulas impone a las viejas ínfulas pedagógicas de llevar orden a todo. 
La formación docente basada en el orden era esperable, razonable y enteramente eficaz cuando el maestro/adulto tenía la posibilidad de ordenar, disciplinar y jerarquizar como parte de una cultura que hacía lo propio fuera de la escuela. Hoy, por el contario, es de esperar una formación docente basada en el conflicto; esto es, dotar a los futuros docentes de herramientas que le sirvan desde el punto de vista actitudinal a sentirse cómodos en el conflicto, a no verlo como una anomalía producto de su inacción o consecuencia de un defecto social subsanable sino como una marca propia de los tiempos y de esta fase del proceso de escolarización.

Desde el punto de vista cognitivo, es necesario dotar a estos futuros docentes de instrumentos conceptuales y metodológicos que les permitan actuar frente a la crisis, sin diagnosticarla como una irregularidad sino como un contexto específico de aprendizaje que, nos guste o no, vino a quedarse en las escuelas, mientras pervivan los rituales y dispositivos posfigurativos de las instituciones escolares modernas.

La segunda consecuencia derivada del mantenimiento de estos ritos y de la reporducción de estos dispositivos es la constatación del carácter asimétrico que, todavía, asumen los vínculos de enseñanza y aprendizaje en las instituciones escolares. Si bien no se habrá de ahondar en los motivos de esta persistencia, llama la atención que la psicología cognitiva asume que esta asimetría parece condición para el aprendizaje (Valsiner, 1994) y especialmente para el aprendizaje escolar (Baquero, 2001).

Pero en el proceso de formación de los futuros docentes se tendrá que apuntar al hecho de que estas asimetrías no son necesarias ni permanentes: su legitimidad es situacional y varía en función de la capacidad de los educadores y de las escuelas de construir escenarios de necesidad de aprendizaje en el que la acción adulta del educador se hace indispensable.

Resulta imposible definir de manera general y abstracta cuáles son los abordajes posibles para instalar asimetrías contingentes que permitan profundizar el rol del educador. Esta pretensión además de infructífera es sumamente peligrosa porque termina por generar en los futuros educadores la vana ilusión de que el proceso escolar puede conducirse mediante la voluntad racional del pedagogo.

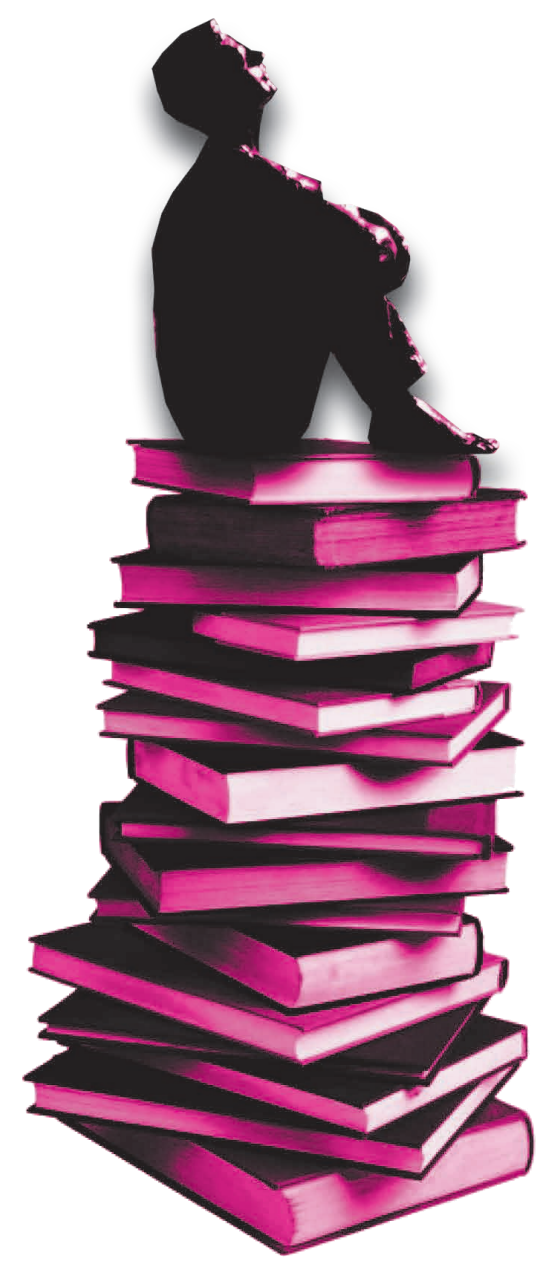


Por el contario, la delimitación de los instrumentos de la práctica es evidentemente situacional y por ende precisa de una capacidad muy flexible, muy plática de interacción con la realidad: la idea de que los alumno van a obedecer a su maestro por el solo hecho de que este ocupa el lugar del que sabe y aquel es el dependiente y heterónomo que solamente precisa aprovechar de la situación de su formación (y que era sancionado por no aprovecharla) funcionaba en épocas posfigurativas, pero ya no encaja con la actual realidad de la escolarización, más mezquina y más reacia a aceptarse como consecuencia pasiva de dictados pedagógicos generales, resultados de racionalidades poco porosas.

Esta contradicción inmanente a la época en la que conviven la necesidad de asimetrías para la acción educadora en un escenario no proclive a su instalación para conformar el sino trágico de la educación escolar de nuestro tiempo: mientras más intentamos instalar asimetrías rígidas y estables, más se desborda de conflictos el escenario y más se consolida la generalización de equivalentes como reacción al intento posfigurativo.

El segundo eje conceptual que interesa aquí desarrollar, algo ya insinuado en párrafos anteriores, tiene que ver con la necesidad de concientizar acerca del peligro por el que transita la profesión docente: si nuestra cultura es de equivalentes generalizados, la necesidad de un otro diferente, con capacidad de trasmitir y legitimidad para hacerlo tiene pronta fecha de vencimiento para convertirse en un mero mediador, un equivalente con una función especializada en la provisión de conocimientos on-demand.

Hace ya varias décadas, Jean Francois Lyotard (1987) alertaba sobre el problema. La condición posmoderna de la cultura alienta respuestas performativas; es decir, basadas en un puro resultado; en el utilitarismo de del ajuste a la resolución concreta de un problema, desmarcándose del peso de la tradición ideológica moderna. Este esquema, llevado al campo de la educación, transforma al docente en un mero proveedor: el peligro no es que los educadores sean sustituidos por máquinas sino que esta sustitución sea apenas la consecuencia de la desaparición de la relación social asimétrica que le daba vida a la función educadora.

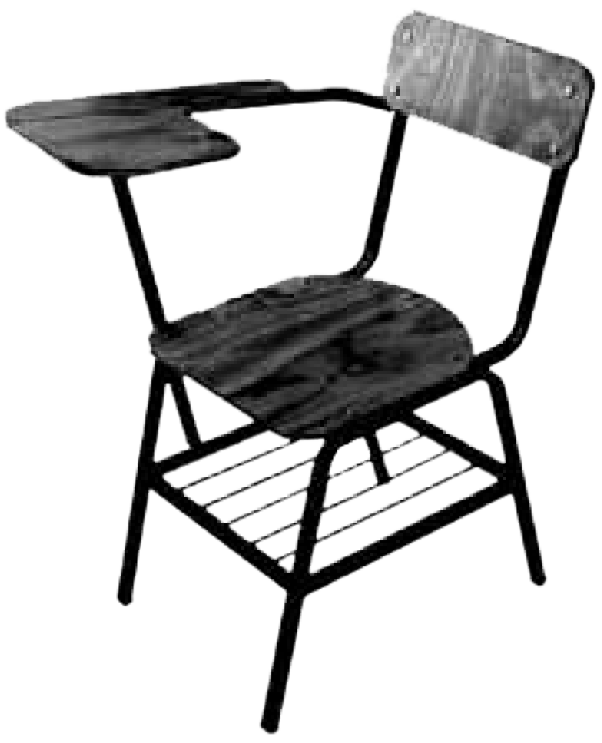

Formar docentes en tiempos prefigurativos supone advertir que su status de educador se construye, y a la vez se juega, en cada oportunidad en que se pueda establecer un vínculo asimétrico y así el docente pueda legitimar su capacidad de ser otro claro y distinto, alguien que tiene algo diferente para dar, alguien a quien se puede recurrir. Para evitar la muerte del profesor que Lyotard (1987) auguraba como corolario de la dilución de las relaciones sociales que históricamente lo generaron, es menester comprender que el ser-educador habrá de comprometerse en cada situación en la que pueda construir su asimetría: una forma de escapar a una desaparición encubierta en una máscara en la que la única función posible es la de un delivery siempre reemplazable. 


\section{Bibliografía}

Baquero, R. (2001). La educabilidad bajo sospecha. Cuaderno de pedagogía rosario. Número 9, Argentina.

Bell, D. (1996). The cultural contradictions of capitalism. New York: Basic Books.

Buckingham, D. (2010). Cultura digital, educacao mediática e o lugar de escolarizacao. Educacao e Realidade. 35(3).

Habermas, J. (1980). Legitimation crisis. London: Heinemann.

Mead, M. (2006). Cultura y compromiso. Barcelona: Gedisa.

Lyotard, J. (1987). La condición posmoderna. Madrid: Cátedra.

Narodowski, M. (1994). Infancia y poder. Buenos Aires: Aique.

Narodowski, M. (1999). Después de clase. Buenos Aires: Novedades Educativas.

Narodowski, M. (2005). “Destinos de la infancia y de los educadores: hiper y desrealización”. En: Germán Arellano Duque. La educación en tiempos débiles e inciertos. Madrid: Antrophos.

Narodowski, M. (2011a). No es fácil ser adulto. Asimetrías y equivalencias en las nuevas infancias y adolescencias. Educación y Pedagogía. Volumen 23, número. 60.

Narodowski, M. (2011b). Papá ¿qué es un adulto? Síntesis Educativa. Disponible en: http://www.sintesis educativa.com. ar/index.php?option=com_content\&view =article\&id=558:papa-que-es-un-adulto\&catid=2:colaboraciones\&ltemid=5

Narodowski, M. y Baquero, R. (1994). ¿Existe la infancia? Revista del Instituto de Investigaciones en Ciencias de la Educación. Número 11.

Sennett, R. (2007). The culture of the new capitalism. New Haven: Yale University Press.

Jaan Valisiner (1994). Co-constructionism and development: a socio-historical tradition. Anuario de Psicología. Número 69.

Yeomans, P. y Dawn, W. (2008). The good, the bad and the wiki: Evaluating student-generated content for collaborative learning. British J ournal of Educational Technology. 39 (6). 